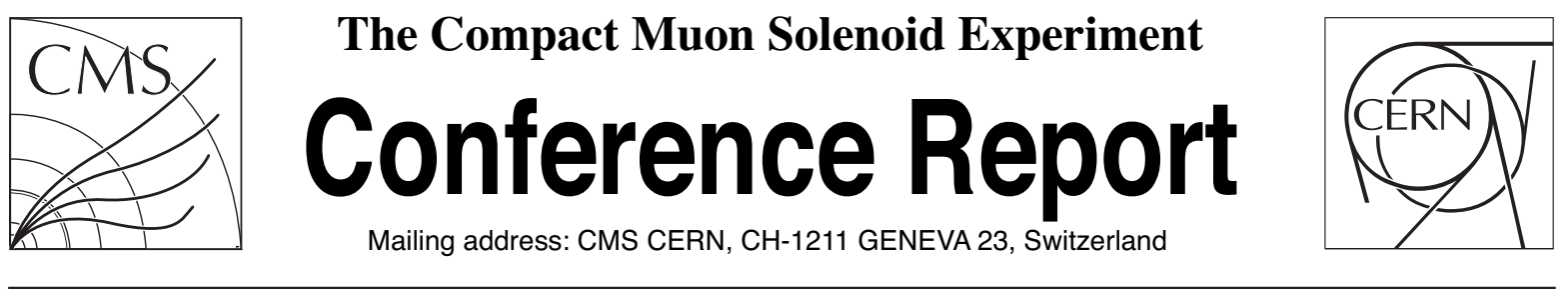

28 November 2014 (v2, 28 December 2014)

\title{
The Data Quality Monitoring Software for the CMS experiment at the LHC
}

\author{
Laura Borrello for the CMS Collaboration
}

\begin{abstract}
The Data Quality Monitoring (DQM) Software is a central tool in the CMS experiment. Its flexibility allows for integration in several key environments Online, for real-time detector monitoring; Offline, for the final, fine-grained Data Certification; Release-Validation, to constantly validate the functionalities and the performance of the reconstruction software; in Monte Carlo productions.Since the end of data taking at a center of mass energy of $8 \mathrm{TeV}$, the environment in which the DQM lives has undergone fundamental changes. In turn, the DQM system has made significant upgrades in many areas to respond to not only the changes in infrastructure, but also the growing specialized needs of the collaboration with an emphasis on more sophisticated methods for evaluating dataquality, as well as advancing the DQM system to provide quality assessments of various Monte Carlo simulations versus data distributions, monitoring changes in physical effects due to modifications of algorithms or framework, and enabling regression modeling for long-term effects for the CMS detector.The central tool to deliver Data Quality information is an interactive web site for browsing data quality histograms (DQMGUI). In this contribution the usage of the DQM Software in the different environments and its integration in the CMS Reconstruction Software Framework (CMSSW) and in all production workflows are presented, with emphasis on recent developments and improvementin advance of the LHC restart at $13 \mathrm{TeV}$. The main technical challenges and the adopted solutions to them will be also discussed with emphasis on functionality and long-term robustness.
\end{abstract}




\title{
The Data Quality Monitoring Software for the CMS experiment at the LHC
}

\author{
L. Borrello for the CMS Collaboration, University of Wisconsin, Madison, WI 53706, USA
}

\begin{abstract}
The Data Quality Monitoring (DQM) Software is a central tool in the CMS experiment. Its flexibility allows for integration in several key environments: online, for real-time detector monitoring; offline, for the final, fine-grained data certification; release validation, to constantly validate the functionalities and the performance of the reconstruction software; in Monte Carlo productions.

Since the end of data taking at a center of mass energy of 8 TeV, the environment in which the DQM lives has undergone fundamental changes. In this contribution the usage of the DQM software in the different environments and its integration in the CMS Reconstruction Software Framework (CMSSW) and in all production workflows are presented, with emphasis on recent developments and improvement in advance of the LHC restart at $13 \mathrm{TeV}$.
\end{abstract}

Index Terms-Detector, data quality monitoring

\section{INTRODUCTION}

$\mathbf{T}$ HE Compact Muon Solenoid (CMS) [1] is a general purpose detector at the Large Hadron Collider (LHC) [2] at CERN. During LHC operation, it is critical for the experiment to have an efficient system which controls the detector working condition and can be used to certify as usable for analysis the recorded data. This task is covered by the Data Quality Monitoring (DQM) framework which is embedded in the CMS software environment (CMSSW) [3]. The DQM system provides a common structure for booking, filling and archiving histograms and scalar monitor elements, called monitor element in the DQM jargon, and for performing automatic quality test. One basic tool of the DQM system is the visualization of the information which is done using an interactive web site (DQM GUI). In addition, tools for data certification are provided for the bookkeeping of information used to define the data sample for physics analysis.

The general structure of the DQM software allows for its integration in several key environments: online, for real-time detector monitoring; offline, for the final, fine-grained data certification; release validation, to constantly validate the functionalities and the performance of the reconstruction software; in Monte Carlo productions.

During the LHC shutdown period, the DQM system has undergone significant changes in many areas to cope with the improvements in the infrastructure and with the needs of the collaboration with an emphasis on more sophisticated methods for evaluating data quality. The DQM system has been also equipped to provide quality assessments of various Monte Carlo simulations versus data distributions and monitoring changes produced by modified algorithms or framework.

This contribution describes the usage of the DQM software in the different environments with an emphasis on recent developments and improvements in preparation of the LHC restart in Run2. The main technical challenges and the adopted solutions will be discussed with emphasis on functionality and long-term robustness.

\section{ONLINE DQM}

The data quality is monitored in real-time during data taking using online DQM applications. Each application receives a fraction of the events with a rate of about $10-15 \mathrm{~Hz}$ to identify problems in the detector and trigger system.

Since the data acquisition system (DAQ) of the CMS experiment has undergone significant changes, the structure of the online DQM has been updated accordingly. During LHC Run 1 , online DQM applications received events from the storage manager proxy over HTTP, while in the new system events are provided in a file per lumi-section, which is the minimum time interval within a run. The software has been updated implementing different tools:

- a daemon to discover new run and start all the DQM applications;

- a new class designed for file discovery and handling of the available data;

- a monitoring system of the online DQM applications based on the elastic-search [4];

- a monitoring system to check the disk space and remove old data files to prevent crashes during data processing.

The daemon controls the status of the available runs and the online DQM applications. When a new run is made available to the DQM system, online applications are stopped and restarted to analyse the live data recorded by the experiment. To cope with possible pauses in the recording of the events, the system has been designed to wait a fixed time and skip any missing file. In order to have an efficient and robust system, status of each application is monitored using a daemon that allows an easy access to event rate, $\log$ file and other information useful to check if the application is properly working.

While the online DQM system is running, histograms and scalar monitor elements are shown in the DQM Graphical User Interface (GUI). Such information is used by shifters which are based at the experimental site as a part of the CMS crew. Before their first shift, shifters are trained by experts to use the tools designed to monitor the detector status. During data taking, shifters have to define the quality of the data registering this information in a specific database and they need to spot problem to minimize the data recorded in non optimal condition. 
An important tool to detect problem is the alarm system which alert shifters on potential issue. It is based on customised histograms defined by subsystem experts to spot critical condition when thresholds are exceeded. This system has been optimised allowing the shift crew to suppress individual alarms once they are acknowledged.

The stability of the DQM online system is constantly monitored by the DQM on-call expert which is available $24 / 7$ to solve all the technical issue and coordinate the activity when a problem is found. Tools for the expert on-call activity has been improved to provide a fast access to information about the system operation and deliver warnings when critical condition are reached.

\section{OfFline And Release VALIDATION DQM}

Offline DQM processes the full data sample recorded by the CMS experiment and produces information needed to define the quality of the data used for physics analyses.

Offline data processing is done at the CERN computing center with a delay of two days to use the updated detector calibration. Information produced in this step includes monitor elements for detectors operation and for physics objects, such as tracks, muons, electrons, jets and missing transverse energy. Once the full run is processed, a file is made available in the offline DQM GUI. Offline DQM files are based on ROOT [5] objects and includes additional information such as the output of quality tests. Information shown in the GUI is finally used by shifters and experts to define the quality of the data. Offline shifts are done in three CMS centers based at CERN, DESY and FNAL site. Shifters have to check a sub-set of monitor elements to decide if the data have to be considered for physics analysis. Data are further checked by detector and physics object experts to correct any mistake done by the shifter and to improve the software and the instructions to produce a more reliable data certification.

Improvements for LHC Run 2 operation have been implemented to be compatible with the new version of the CMS reconstruction software which is now based on new technologies such as multi-core and multi-thread processing mode [6]. Such improvements are used also in the release validation process which is needed to scrutinize and test changes in the software reconstruction algorithms and in the detector conditions. For each new release, recorded and simulated data are processed with the CMS software and the same applications used for the offline DQM are used to monitor the performance of the detector and physics objects. As in the offline DQM workflow, information is shown in the DQM GUI to be checked by release validation experts which provide feedback on the status of the release. This task is fundamental for the experiment since any update in the software has to be validated before the deployment in the official release for a reliable and efficient data processing.

\section{The GraphicAl USER INTERFACE (GUI)}

The DQM GUI is the central tool of the data quality monitoring system and it is used for all steps: online, offline, release validation and Monte Carlo production.
It is a web application that collects, stores and displays data quality information shown as histograms and scalar monitor elements, and can be accessed worldwide by authenticated users.

Each DQM workflow share the same code to display the information for shifters and experts investigation but the user interface is varying to match specific needs.

Figure 1 shows a schematic view of the summary page of the online GUI where the status for each CMS detectors is shown. The content of the GUI is organized to cover different tasks

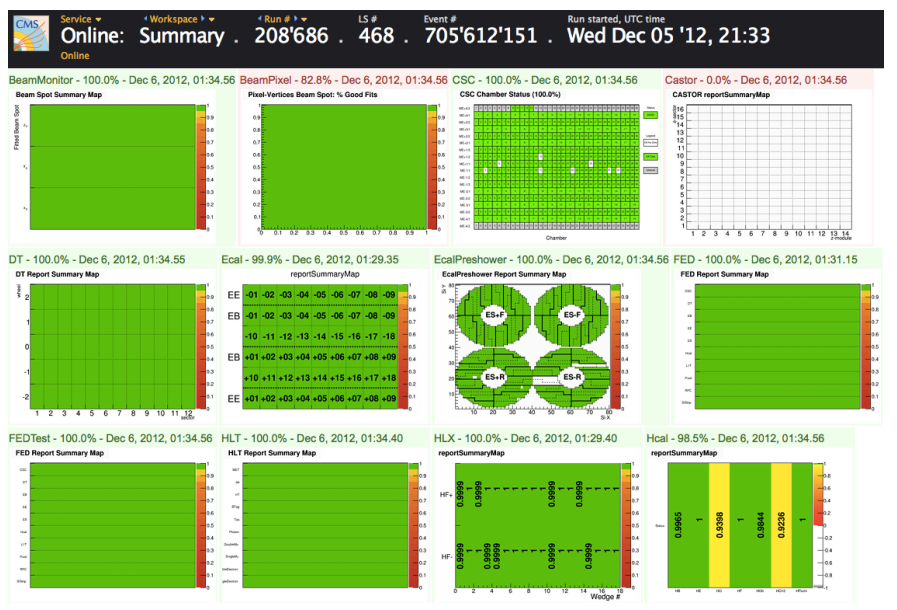

Fig. 1. Snapshot of the CMS Graphical User Interface used for the visualisation of the data quality information.

and includes high-level summaries, shift views and expert areas which are displayed with a common histogram style editor. Within a workspace, histograms can be grouped into common layouts to provide a simple and fast access to define data quality. This flexibility is used by experts to optimize the information of different DQM workflow and provide a simple starting point for the data quality monitoring.

During LHC long shutdown, some changes have been planned to update the DQM GUI:

- underlying schema-evolution of the back-end to allow to reliably host more data while keeping the same performances;

- modify the current back-end to be a distributed back-end for a better scalability and to use open-source software instead of ad-hoc $\mathrm{C}++$ back-end improving the maintainability.

This changes will further improve the performance of the tool which has been extensively tested since 2008 and proved to be very efficient and robust.

\section{Data Certification}

Data recorded by the CMS experiment have to be validated before their use is approved for physics analyses. Certification procedure includes three different steps:

- offline shifters define a flag (good or bad) for each detector, trigger and physics object, such as tracks, muon, electron, jets and missing transverse energy; 
- detector and physics object experts check the flags and provide additional information about the reason for the data to be considered as bad;

- DQM experts create a JSON file specifying the list of runs and lumi-sections to be used for physics analyses.

Data quality information is stored in a specific database called Run Registry [6] which has a front-end web application. A schematic view of the Run Registry is shown in figure 2. Database is accessed regularly by online and offline shifters

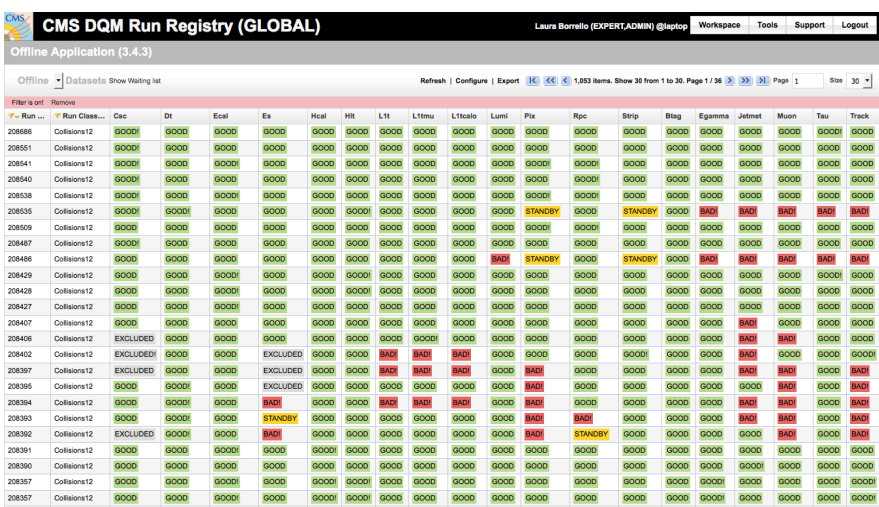

Fig. 2. Snapshot of the CMS run database for the data quality information.

to bookkeep the status of the data certification in a central workspace. Details about the quality of recorded data can be corrected if any mismatch is found by experts. The granularity considered to store the information is the lumi-section, which is the minimum time interval within a run, in order to reduce the amount of data lost due to short problematic condition within a run.

During LHC Run 1, the certification procedure included some manual steps done to include the feedback from certification experts. This manual step were particularly error prone and in some cases required the information in the database to be corrected in a later step.

Main improvement introduced in the data certification procedure is the automation of manual operations reducing the risk of miscommunication and human caused errors. This aim has been fulfilled using the full functionality of the Run Registry application since each detector, trigger and physics object group has its own local workspace. Data certification experts fill the information in their own workspace adding a cause for each specific problem found in the recorded data. When the previous steps are completed, final certification is done using a global workspace where the results filled in local workspace are automatically copied with a synchronisation tool. This step is used to update the information about the certification status such as the quality flag, the comment and the reason for the data to be considered bad. Once the run certification is complete and consistent, run database is accessed via API to produce a file in the JSON format delivered to the CMS collaboration for the physics analyses.

\section{READINESS OF THE DQM SYSTEM FOR LHC RUN 2}

Since June 2014 the CMS experiment is organizing short periods of cosmic data taking to monitor the performance of the detector after the intervention and the changes done during the LHC shutdown period.

Improvements implemented for the Data Quality Monitoring system are currently under test to verify that the expected performance are met.

Results from current data taking are satisfactory. Online and Offline DQM proved to be stable and effective in monitoring the detector and physics object performance. Release validation is working fine to verify the validity of software changes and updated condition in the database. Data certification proved to be more reliable and robust since most of the manual steps have been replaced with a fully automatic procedure.

The outcome of all the tests done until now are confirming the excellent work done to ensure optimal performance for monitoring the CMS experiment operation during the LHC Run 2.

\section{REFERENCES}

[1] The CMS Collaboration, The CMS Experiment at the CERN LHC, JINST $3 \mathrm{~S} 08004,2008$

[2] LHC Machine, JINST 3 S08001, 2008.

[3] The CMS Collaboration, CMS Physics Technical Design Report Volume I, Detector Performance and Software, CERN/LHCC/2006- 001, CMS TDR 8.1, 2 February 2006

[4] http://www.elasticsearch.org/

[5] ROOT - A data analysis framework, http://root.cern.ch

[6] C. Grandi et al.,CMS computing model evolution, Journal of Physics: Conference Series 513 (2014) 032039

[7] V. Rapsevicius, CMS Run Registry: Data certification bookkeeping and publication system, Journal of Physics Conference Series (2011). 331 042038 